\title{
L'enseignement des traités autochtones dans une classe d'immersion française. L'apprentissage des thèmes reliés à la réconciliation dans un contexte français minoritaire
}

\author{
Naomi Fortier-Fréçon et Leia Laing
}

Volume 31, numéro 1, 2019

L’autochtonisation pour préparer un avenir commun

URI : https://id.erudit.org/iderudit/1059128ar

DOI : https://doi.org/10.7202/1059128ar

Aller au sommaire du numéro

Éditeur(s)

Presses universitaires de Saint-Boniface (PUSB)

ISSN

0843-9559 (imprimé)

1916-7792 (numérique)

Découvrir la revue

Citer cet article

Fortier-Fréçon, N. \& Laing, L. (2019). L'enseignement des traités autochtones dans une classe d'immersion française. L'apprentissage des thèmes reliés à la réconciliation dans un contexte français minoritaire. Cahiers franco-canadiens de l'Ouest, 31(1), 109-125. https://doi.org/10.7202/1059128ar
Résumé de l'article

L'intégration des thèmes reliés à la réconciliation tel que demandé dans les appels à l'action de la Commission de vérité et réconciliation du Canada dans les cours de langue française permet aux élèves d'analyser les relations de pouvoir du discours normatif qui favorise le privilège blanc. Cependant, pour les élèves qui vivent dans un contexte où le français est la langue de la minorité, il y a aussi le défi d'avoir accès à des ressources appropriées et des opportunités d'utiliser la langue française à l'extérieur du contexte scolaire. Cette réalité peut favoriser une difficulté au niveau de la compréhension de thèmes complexes tels que la discrimination et le racisme. Cette situation peut avoir pour effet de complexifier l'apprentissage de la langue seconde. L'approche multidisciplinaire s'avère être une avenue pour encourager le développement langagier des élèves. Cet article relate une expérience menée auprès d'élèves du secondaire inscrit dans un programme d'immersion française dans le cadre de leur cours de français art langagier et de leurs cours de sciences sociales. L'expérience que nous avons vécue avec nos élèves dans le cadre de ce projet éducatif nous permet d'identifier ce qui contribue à transformer les attitudes et à encourager le changement social au sujet d'une meilleure compréhension des enjeux reliés à la culture et aux traditions autochtones au Canada tout en favorisant le développement de la langue française.
Tous droits réservés (C Centre d'études franco-canadiennes de l'Ouest (CEFCO) et Presses universitaires de Saint-Boniface (PUSB), 2019
Ce document est protégé par la loi sur le droit d'auteur. L’utilisation des services d'Érudit (y compris la reproduction) est assujettie à sa politique d'utilisation que vous pouvez consulter en ligne.

https://apropos.erudit.org/fr/usagers/politique-dutilisation/ 


\title{
L'enseignement des traités autochtones dans une classe d'immersion française. L'apprentissage des thèmes reliés à la réconciliation dans un contexte français minoritaire
}

\author{
Naomi FORTIER-FRÉÇON et Leia LAING
}

\section{RÉSUMÉ}

L'intégration des thèmes reliés à la réconciliation tel que demandé dans les appels à l'action de la Commission de vérité et réconciliation du Canada dans les cours de langue française permet aux élèves d'analyser les relations de pouvoir du discours normatif qui favorise le privilège blanc. Cependant, pour les élèves qui vivent dans un contexte où le français est la langue de la minorité, il y a aussi le défi d'avoir accès à des ressources appropriées et des opportunités d'utiliser la langue française à l'extérieur du contexte scolaire. Cette réalité peut favoriser une difficulté au niveau de la compréhension de thèmes complexes tels que la discrimination et le racisme. Cette situation peut avoir pour effet de complexifier l'apprentissage de la langue seconde. L'approche multidisciplinaire s'avère être une avenue pour encourager le développement langagier des élèves. Cet article relate une expérience menée auprès d'élèves du secondaire inscrit dans un programme d'immersion française dans le cadre de leur cours de français art langagier et de leurs cours de sciences sociales. L'expérience que nous avons vécue avec nos élèves dans le cadre de ce projet éducatif nous permet d'identifier ce qui contribue à transformer les attitudes et à encourager le changement social au sujet d'une meilleure compréhension des enjeux reliés à la culture et aux traditions autochtones au Canada tout en favorisant le développement de la langue française.

Mots-clés: enseignement, traités, peuples autochtones, réconciliation, contexte francophone minoritaire 
$\mathrm{Au}$ Canada, le programme d'immersion permet aux élèves d'apprendre la langue française à travers l'étude de différentes matières scolaires. Dans le contexte de l'apprentissage d'une langue seconde ou additionnelle, l'étude de thèmes reliés à la justice sociale peut s'avérer un défi au niveau de la compréhension d'idées complexes et l'accessibilité à des ressources pédagogiques appropriées au niveau de langue des élèves ${ }^{1}$. Devant le double défi, celui de développer une compréhensiondes concepts enseignés dans le contexte de l'apprentissage d'une langue seconde ou additionnelle (Lyster, 2017, 2007), il était important pour nous de favoriser des stratégies pédagogiques qui pouvaient nous permettre de favoriser l'inclusion de thèmes complexes, tels que la discrimination et le racisme.

Nous avons constaté que nos élèves étaient en mesure de comprendre les idées qui étaient exprimées à l'oral ainsi que de les partager de façon verbale. Cependant, des difficultés importantes survenaient au niveau de la production écrite. Les élèves semblaient être en mesure de partager plus facilement leurs idées par le biais de projets artistiques que sous la forme écrite.

Notre second défi fut d'avoir accès à des ressources pédagogiques adaptées à l'enseignement dans le contexte où le français était à la fois la langue minoritaire ainsi que la langue seconde ou additionnelle de l'élève. À l'époque où nous avons travaillé à la mise en place de ce projet éducatif (20142015), les conversations au sujet de la présence d'un racisme systémique commençaient à être présentes chez nos collègues anglophones mais très peu du côté francophone et encore moins dans le contexte de l'apprentissage d'une langue seconde. En conséquence, les ressources pédagogiques en français étaient très limitées au niveau du nombre et celles qui étaient disponibles s'avéraient, la majorité du temps, non adaptées à la réalité vécue dans le contexte de l'immersion française. Sachant que l'apprentissage des thèmes reliés à la réconciliation et aux traités est essentiel pour nos élèves, nous ne pouvions nous résoudre à passer outre ce sujet. Un projet éducatif pour répondre à ces attentes a donc été mis en place.

\section{Le contexte de notre projet éducatif}


La province de la Saskatchewan se situe sur les terres des peuples Cris, Saulteaux, Assiniboine, Dene, Dakota et la terre ancestrale du peuple Métis (Office of the Treaty Commissioner, 2014). L'histoire canadienne raconte que vers la fin du XIX siècle, l'extermination du bison par les colonisateurs européens fut une situation difficile à vivre, car le bison était un élément important du mode de vie des peuples autochtones dans les Prairies (Daschuk, 2013). L'arrivée de l'industrialisation a aussi provoqué des changements dans les modes de vie traditionnels. En conséquence, les peuples Cris, Saulteaux, Assiniboine et Dene ont accepté de signer les traités numérotés avec le gouvernement canadien qui agissait à cette époque à titre de représentant de la couronne britannique. Ces ententes formelles représentent les accords juridiques qui définissent, encore aujourd'hui, les termes qui régissent le partage du territoire et des ressources de la province de la Saskatchewan. La signature des traités a notamment permis aux immigrants d'origine européenne et nord-américaine de s'installer sur les terres agricoles des Prairies canadiennes à très bon prix. Aujourd'hui, l'ensemble du territoire de la Saskatchewan est divisé en sept traités numérotés (traités $\mathrm{n}^{\circ}$ 2, 4, 5, 6, 7, 8, 10). Depuis 2007, le gouvernement de la Saskatchewan a rendu obligatoire l'enseignement des traités de la maternelle à la $12^{\mathrm{e}}$ année (Saskatchewan Education, 2013). L'intégration de la perspective autochtone dans les programmes d'études a pour but de répondre à quatre attentes:

- Favoriser le développement des connaissances au sujet de la présence d'une relation entre les traités et l'identité des peuples autochtones (langue, traditions, vision du monde);

- Développer une compréhension au sujet de l'esprit et du sens des traités afin de favoriser un lien entre les pensées et les actions;

- Prendre conscience de l'impact du contexte historique aux niveaux social, culturel, économique et politique dans le passé et dans la vie quotidienne de l'élève;

- Reconnaître la nature sacrée de cette entente qui a pour but d'assurer le bien-être de tous les habitants qui partagent le même territoire. 
Ce projet éducatif a été réalisé dans une école secondaire située dans un milieu urbain sur le territoire du Traité $\mathrm{n}^{\circ} 4$. Au moment de la réalisation de ce projet, la programmation de cette école la $9 \mathrm{e}$ à la $12^{\mathrm{e}}$ incluait le programme d'immersion française, d'anglais, du baccalauréat international et des programmes en éducation alternative. Les étudiants de cette école étaient principalement de descendants de peuples colonisateurs, ainsi que d'immigrants de première et seconde générations, avec un petit nombre d'élèves s'identifiant comme Premières Nations ou Métis. Cette année-là, un des objectifs de notre école était de promouvoir une pratique pédagogique qui reflétait un choix de ressources qui favorisait une ouverture culturelle diversifiée.

\section{Projet Traité $n^{\circ} 4$ : La prochaine génération}

Le projet éducatif que nous avons réalisé avec nos élèves avait pour but de permettre à nos élèves d'explorer l'idée d'une appartenance au Traité $n^{\circ} 4$ afin de leur permettre de comprendre le rôle de leur génération dans le développement d'un environnement plus juste et ouvert. Utilisant comme point de départ les idées reliées à l'établissement d'une relation mutuelle avec l'endroit où ils habitent et ses premiers habitants, notre désir était d'offrir aux élèves l'occasion de prendre conscience de la présence de situations oppressives invisibles dans leur environnement, tel que cela a déjà été souligné par McIntosh, (1990) et Laroque, (2016) ainsi que de leur permettre d'analyser les relations de pouvoir du discours normatif qui favorise le privilège blanc relevé par Pete et al., (2013).

Ce projet éducatif résulte d'une prise de conscience que nous avons faite en observant nos salles de classe et en discutant avec nos élèves. Avant le début de ce projet, nous avions remarqué que l'histoire de la Saskatchewan était racontée principalement selon la perspective européenne des pionniers et que l'histoire des peuples autochtones était absente. Sachant que le programme d'étude indique qu'à la fin de la $12^{\mathrm{e}}$ année l'élève soit en mesure de comprendre l'importance des traités, il était impératif que ces thèmes soient inclus dans notre enseignement. Aussi, il convient de prendre en considération les 94 appels à l'action du rapport final Honorer la vérité, réconcilier pour l'avenir (Commission Vérité et Réconciliation, 2015). Créé dans le but de documenter la difficile réalité vécue dans les écoles résidentielles, la publication des appels à l'action a pour 
but d'encourager la mise en place d'un dialogue qui supporte le processus de réconciliation entre Autochtones et les Allochtones au Canada. Les appels à l'action n ${ }^{\circ} 62$ et n ${ }^{\circ} 63$ parlent de:

62.i. rendre obligatoire, pour les élèves de la maternelle à la douzième année, l'établissement d'un programme adapté à l'âge des élèves portant sur les pensionnats, les traités, de même que les contributions passées et contemporaines des peuples autochtones à l'histoire du Canada;

63.i. l'élaboration et la mise en œuvre, de la maternelle à la douzième année, de programmes d'études et de ressources d'apprentissage sur les peuples autochtones dans l'histoire du Canada, et sur l'histoire et les séquelles des pensionnats.

Ces attentes nous ont confirmé qu'il était important de permettre à nos élèves d'apprendre d'avantage à ce sujet. Pour ce faire, nous avons choisi d'ouvrir nos salles de classe afin de permettre à des membres des communautés des Premières Nations, métisse et universitaire d'échanger avec nos élèves. Les stratégies pédagogiques valorisées ont été l'histoire orale, l'analyse de documents historiques, le développement de la pensée critique au sujet de la notion de l'existence de privilèges et d'une responsabilité sociale d'une société envers ses membres ainsi que l'utilisation de l'art visuel comme médium d'expression des idées.

\section{L'expérience initiale: (2014-2015)}

Notre objectif avait pour but de permettre à nos élèves d'immersion française de sortir de la salle de classe et d'aller rencontrer des élèves du même groupe d'âge qui fréquentaient lesécoles anglophones environnantes dont la majorité sont à forte proportion autochtone. Nous avions remarqué que même si nos écoles appartenaient à la même commission scolaire, très peu de contacts sont possibles entreles écoles. En conséquence, lesécoles ont pris l'habitude d'évoluer en vase clos indépendamment les unes des autres. En discutant avec nos élèves, nous avons constaté que ces derniers avaient très peu de connaissances au sujet de la vie scolaire à l'extérieur des murs de notre école. Encore plus inquiétant, les connaissances exprimées étaient plutôt de nature stéréotypée et encourageaient la diffusion de préjugés et d'idées préconçues. Ainsi, notre objectif n'était pas 
seulement d'acquérir de nouvelles connaissances mais aussi d'avoir l'occasion de les mettre en pratique en favorisant le début d'un dialogue qui encouragerait le respect et des valeurs d'inclusion.

Afin de favoriser une pédagogie qui encourage la décolonisation du discours éducatif, il était important que nos élèves puissent vivre une expérience culturelle qui leur permettrait de prendre connaissance des témoignages et des perspectives autochtones authentiques (Weenie, 2014; Tuck \& Yang, 2012). Ainsi, il fut primordial pour nous de commencer par établir des relations avec des membres des groupes autochtones qui résidaient sur le territoire du Traité $n^{\circ} 4$. Pour ce faire, nous avons communiqué avec le coordonnateur de l'éducation autochtone de notre commission scolaire qui nous a permis d'établir un lien avec un ainé Nehiyaw (Cri des Plaines) et d'autres membres de la communauté culturelle des Cris des Plaines. Un lien a aussi été établi avec deux professeurs de l'Université des Premières Nations du Canada et l'artiste Cri/ Métis Ray Keighley. Lors de la première rencontre avec ces partenaires, un comité de référence a été créé dans le but d'offrir à ce projet un soutien éducatif et culturel. Ainsi, ce comité avait pour but de partager des recommandations afin qu'une vision du monde qui inclut les perspectives des Premières nations et des Métis en Saskatchewan soit incluse dans toutes les étapes de ce projet. L'établissement de relations, d'échanges avec les membres de la communauté des Cris des Plaines et l'inclusion de cérémonies traditionnelles, telles qu'une cérémonie de purification et une cérémonie du calumet (Wilson, 2001) furent très importantes dans la réalisation de ce projet éducatif, car ces initiatives nous ont permis d'inclure les voix autochtones de façon respectueuse.

Au fil de nos conversations, nous avons constaté qu'il y avait un grand manque de connaissances générales envers les peuples des Premières Nations et des Métis auprès de nos élèves. L'idée d'inviter des conférenciers est venue de ce besoin $d$ 'informer les élèves d'une manière plus authentique que possible. Lorsque le comité discutait de thèmes possibles, nous nous sommes rapidement rendu compte qu'il était nécessaire de ne pas victimiser ni de présenter des images uniquement historiques et stéréotypées. Pour cette raison, l'idée d'inviter 
un grand nombre de conférenciers qui pourraient aborder une variété de sujets s'est avérée la meilleure option. Nos intentions se sont manifestées sous forme d'une conférence jeunesse, ayant pour thème l'exploration d'une appartenance au Traité $n^{\circ} 4$. La rencontre entre les élèves de la $9^{e}$ à la $12^{e}$ année provenant de quatre écoles secondaires, réalisé à l'Université des Premières Nations du Canada, a permis de favoriser cette compréhension par la création d'une murale représentant les idées des participants. Cette murale est aujourd'hui exposée au centre Mâmawêyatitân, à Regina. Dès le début de ce projet, il était important pour nous que cette murale soit exposée dans un lieu qui permet que les idées de nos élèves se répandent à l'extérieur de la salle de classe afin de dialoguer avec un plus grand public.

\section{Le projet réédité (2016-2018)}

L'année suivante, un nouveau groupe d'élèves provenant de plusieurs écoles secondaires a eu l'occasion de se réunir. La création d'un livre électronique leur a permis d'exprimer leur compréhension desidées présentéeslors dela secondeconférence jeunesse. Lors de la troisième année, notre projet pédagogique a inclus des élèves de l'élémentaire. Sur une période de deux ans, des élèves de la $5^{\mathrm{e}}$ à la $8^{\mathrm{e}}$ année ont travaillé avec l'artiste Cri-Ojibwé Lloyd Dubois sur la construction d'une murale qui représentait leurs idées face à une appartenance au Traité $n^{\circ} 4$. À cette occasion, les élèves d'une école d'immersion et d'une école francophone ont eu la possibilité de se rencontrer et de commencer à établir des liens d'amitié entre les deux groupes. Nous croyons que ces rencontres entre les élèves provenant d'écoles différentes favorisent l'exploration de nouvelles perspectives, car elles exigent que ces derniers sortent de leurs zones de confort. Cette action d'aller vers l'autre physiquement permet d'établir les bases d'un dialogue puisque la réalité est vécue de façon concrète. Ainsi, ce qui pourrait être perçu de façon négative est confronté à quelque chose de tangible. À long terme, nous croyons que cette rencontre entre différents groupes pourrait encourager l'établissement d'idées et de croyances qui appuient les valeurs d'inclusion et de respect. De plus, pour les élèves d'immersion française, cette rencontre leur a aussi permis d'être de communiquer avec des membres de 
la communauté francophone ce qui s'avère important dans le contexte où la langue française est minoritaire.

De retour en classe, nous élèves ont indiqué avoir compris qu'être membres d'une société où leurs actions et leurs pensées avaient des conséquences dans la vie des autres. Ils nous ont aussi exprimé avoir commencé à comprendre que la présence d'un sentiment d'empathie et d'un désir d'apprendre ne leur permet pas nécessairement de changer leur vision du monde. Cela pourrait expliquer pourquoi ils sont en mesure de fournir des réponses jugées correctes dans les questions touchant la réconciliation et, en même temps, ne pas être en mesure de mettre en pratique dans leur vie quotidienne ce qu'ils ont appris en salle de classe. Par conséquent, comme le précise Simpson (2001), nous croyons non seulement qu'il ne suffit pas de dire que nous sommes contre la propagation des idées injustes mais aussi de comprendre que le respect de la dignité humaine exige que des mesures concrètes soient prises pour favoriser la présence d'un changement social qui encourage des valeurs de justice et d'équité reliées à la réconciliation entre les peuples autochtones et allochtones ${ }^{2}$.

\section{Le double défi de l'apprentissage dans un contexte français minoritaire}

Comme nous l'avons mentionné précédemment, notre désir était d'encourager à la fois l'inclusion des idées reliées à la justice sociale ainsi que le développement langagier de nos élèves. En milieu minoritaire, lorsque l'élève anglophone sort de la classe, toutes ses interactions se déroulent en anglais. Ainsi, nous avons éprouvé au départ quelques difficultés à répondre aux attentes langagières du programme d'étude du cours Français Art Langagier, car le temps pour s'exprimer à l'oral, à l'écrit et les périodes de lecture en français est limité. Il était donc primordial de maximiser toutes les occasions. Malgré nos efforts de trouver des aînés qui s'exprimaient en français, les gens des communautés autochtones situées sur le territoire du Traité $n^{\circ} 4$ s'expriment majoritairement en anglais. Cette situation n'est pas surprenante puisque l'anglais est la langue de la majorité en Saskatchewan, tandis que le français peut représenter une forme de traumatisme car selon les témoignages que nous avons entendus, elle a été imposée aux enfants dans certains pensionnats autochtones. Aussi, nous 
avons cru nécessaire d'établir un dialogue avec nos collègues anglophones afin de favoriser un lien entre nos salles de classe et les communautés autochtones établies sur le territoire du Traité $n^{0} 4$ qui ont plus d'expériences dans le domaine de la justice sociale. Cependant, il nous a fallu trouver une solution qui nous permettrait d'en apprendre davantage au sujet des traités autochtones dans leur cours de français. Ainsi, notre objectif a été de favoriser l'utilisation de la langue française lors des périodes d'enseignement dans la salle de classe tout en permettant à nos élèves d'entrer en contact avec des membres des communautés autochtones qui s'exprimaient majoritairement en anglais. Ces derniers ont partagé leur vécu selon la tradition orale des histoires au sujet de leurs communautés et de leurs visions du monde. En conséquence, notre désir a été de créer un pont entre les ressources anglophones et l'enseignement des traités dans un contexte français minoritaire.

À première vue, notre impression a été que l'utilisation de l'anglais pourrait aider nos élèves à comprendre plus facilement l'étude de thèmes complexes (Cummins, 2014; Swain \& Lapkin, 2013). Cependant, le fait que nos écoles soient situées dans une région où la langue française est vécue dans un contexte minoritaire a eu pour effet que l'anglais s'est rapidement imposé comme lingua franca (Steyaert, Ostendorp, Gaibrois, 2010), et ce, même si cette dernière n'est pas la langue maternelle de nos conseillers autochtones. À ce sujet, il convient de préciser que la langue anglaise est non seulement la langue majoritaire au Canada mais aussi celle qui a le plus de pouvoir au niveau mondial (Ballinger, Lyster, Sterzuk et Genesee, 2017; Landry et Allard, 2016). Ainsi, le fait que la langue française en milieu minoritaire côtoie la langue de la majorité ne serait pas sans conséquence puisque, selon Vézina et Houle (2014), il est important de prendre en considération l'impact des caractéristiques sociales présentes dans le milieu de vie des élèves. En effet, dans un contexte où le français n'est pas la langue de discussion à la maison et qu'elle n'est pas valorisée lors des échanges sociaux avec les autres écoles, nous avons malheureusement constaté une diminution de son utilisation pendant les cours de français art langagier.

De plus, tel que mentionné par Landry (2015), il nous a fallu prendre en considération la présence de vécus enculturants. 
Ces derniers sont associés au processus de socio-construction des idées qui favoriseraient la reproduction inconsciente des normes sociales valorisées par la majorité. Dans le contexte des programmes d'immersion française, l'anglais est la langue de la majorité de nos élèves, et le français représente la langue d'usage pour réussir ce programme scolaire. En tant qu'enseignantes francophones, nous avons remarqué que l'inclusion de la langue anglaise dans ce projet éducatif a favorisé la reproduction inconsciente des valeurs linguistiques présentées par les membres anglophones qui ont participé à ce projet, ce qui a eu pour effet de limiter la présence de la langue française. La présence de la langue anglaise n'était pas mauvaise en soi puisqu'elle nous permettait d'atteindre les objectifs que nous nous étions fixés au niveau des idées reliés à la justice sociale. Cependant, nous avons remarqué que pendant ce projet éducatif, initialement conçu pour répondre aux besoins des élèves d'immersion française, a été mis en œuvre en anglais.

La présence croissante de la langue anglaise dans notre projet éducatif nous a permis de réfléchir aux «perceptions du statut et de la légitimité des groupes linguistiques» (Landry, 2015, p. 74). Ainsi, tel que démontré dans le modèle de l'autodétermination collective (Landry 2008, 2009), nous avons clairement ressenti la présence de deux forces qui s'opposaient l'une à l'autre. La présence de la langue anglaise favorisait le contrôle du capital linguistique de ce projet éducatif. En tant qu'enseignantes francophones dans un programme d'immersion française, nous avons aussi remarqué la présence d'une négociation au niveau de notre identité linguistique. Comme membre du groupe minoritaire, nous souhaitions favoriser une conscientisation sociale dans le but de faire valoir l'affirmation et la légitimité de notre identité linguistique ainsi que notre vision du monde francophone. Cette réalité a eu un effet de découragement puisque, malgré un fort désir d'affirmation, nous avions constamment l'impression que la langue dominante était l'anglais, et qu'elle avait une priorité face au français qui était la langue d'enseignement de notre programme d'étude. Cependant, notre désir de vouloir créer de l'espace pour la langue française, nous a permis de prendre conscience qu'il y avait d'autres langues qui étaient dans une situation minoritaire, notamment le Cri et la langue Mitchif. 
Cette prise de conscience en tant qu'enseignantes d'immersion française nous a permis de constater la présence de «deux discours de légitimité en opposition» (Landry, 2015, p. 61). Sans contredit, la langue anglaise possédait une légitimité culturelle puisqu'elle avait le pouvoir de s'imposer dans un projet éducatif conçue pour des élèves d'immersion française. En même temps, lorsque nous étions avec des locuteurs francoparlants, l'utilisation de la langue française était présente ce qui nous a permis de constater, tel que précisé par Landry (2015), la présence d'un discours de solidarité envers la langue française. Notre inquiétude face à la présence croissante de la langue anglaise dans ce projet éducatif était, comme le précise Skutnabb-Kangas (2002), qu'à long terme les limites vécues au niveau de l'utilisation de la langue française entre les participants provoquent une absence de légitimité, ce qui aurait pour effet de limiter le temps accordé à la langue française et le nombre d'élèves qui choisissent de s'exprimer dans la langue de la minorité. Aussi, nous avons constaté que la présence d'une limite au niveau de l'utilisation de la langue française pouvait à long terme favoriser une modification de notre identité linguistiquem puisque nous n'avions pas la possibilité d'utiliser la langue française sur une base régulière pendant nos interactions avec les autres enseignants (Von Staden \& Sterzuk, 2017), et une tendance à utiliser moins fréquemment la langue française pour exprimer nos idées lors des rencontres avec les enseignants des écoles anglophones (Ballinger, Lyster, Sterzuk \& Genesse; 2017).

À ce sujet, Bourdieu (2001) précise que «les échanges linguistiques sont aussi des rapports de pouvoir symbolique où s'actualisent les rapports de force entre les locuteurs ou leurs groupes respectifs» (p.59-60). Le pouvoir symbolique défini par Bourdieu ne se limiterait donc pas à une seule forme de pouvoir mais inclurait plusieurs formes qui lorsque reconnues sont considérées comme étant légitimes. En conséquence, «le poids des différents agents dépend de leur capital symbolique, c'està-dire de la reconnaissance, institutionnalisée ou non, qu'ils reçoivent d'un groupe» (Bourdieu, 2001, p. 107).

En situation minoritaire, la possibilité d'offrir à nos élèves des situations d'apprentissage peut être contraignante puisqu'elle ne nous permet pas d'accéder facilement à des 
capitaux linguistiques en français (Bourdieu, 2001). Cette situation est contradictoire puisque le but avoué du programme d'immersion française est de favoriser l'obtention d'un capital linguistique lorsque l'élève est en mesure de s'exprimer dans les deux langues officielles du Canada. Cependant, dans le contexte d'une école d'immersion française nous avons remarqué que nos élèves avaient tendance à s'exprimer dans la langue de la majorité au détriment du français langue seconde/additionnelle perçue comme étant socialement dévalorisée.

Au niveau de l'apprentissage d'une langue seconde ou additionnelle, Boutouchent (2016) précise que «le contexte francophone minoritaire semble influencer autant les francophones que les francophiles» (p.103). En conséquence, les conditions qui affectent les francophones vivant en milieu minoritaire au niveau de la sauvegarde de la langue française auraient un effet similaire pour les anglophones pendant leur processus d'apprentissage puisque leur exposition à la langue et la culture francophone serait plus limitée. D'où l'importance d'offrir des occasions à nos élèves d'immersion française, qui étudient dans un contexte minoritaire, d'être exposés le plus possible à la langue française.

C'est dans ce contexte que notre expertise d'enseignement dans le programme d'immersion française a pris toute sa signification, puisqu'il nous fallait trouver une façon de sauvegarder l'espace réduit qui était accordé à la langue française. Pour ce faire, nous avons commencé en prenant le temps de discuter avec nos élèves de la réalité vécue lorsque la langue française est vécue dans un contexte minoritaire. La majorité de nos élèves nous ont avoué ne pas être au courant de cette réalité linguistique. Le but avoué de cette démarche n'était pas de détourner notre regard de notre objectif principal, celui de vouloir apprendre davantage au sujet des traités autochtones. Cependant, pour réaliser cet objectif dans une langue seconde/ additionnelle, il nous apparaît essentiel que l'élève d'immersion comprenne que son apprentissage de la langue française s'insère dans un contexte politique où les luttes de pouvoir favorisent un groupe au détriment de l'autre. Comme le précise Allard, Landry et Deveau (2005), nous croyons que notre rôle en milieu minoritaire ne se limite pas à l'enseignement de la langue et/ou à l'enseignement des sujets académiques, mais aussi à prendre 
le temps d'enseigner au sujet des contextes historique, social, politique et culturel dans lequel évolue le programme d'étude que nous avons la responsabilité d'enseigner.

\section{Nous appartenons tous aux traités}

Au début du projet, nous n'avions pas pris conscience de l'ampleur de notre voix. Aujourd'hui, nous prenons conscience que les choix de nos paroles et de nos mots dénotent la présence de privilège. Dans notre cas, nos idées ne sont nouvelles; elles n'ont certainement pas commencé avec nous. De nombreux Autochtones réclament leurs droits et dénoncent les injustices depuis des décennies, mais leurs voix ne sont pas toujours entendues par ceux qui ont un pouvoir ou une influence. L'aîné Nehiyaw avec qui nous avons eu le privilège de travailler en collaboration, Lifespeaker Noel Starblanket, nous a fait remarquer un jour que, depuis le début de sa carrière politique, il travaille à faire avancer les idées reliées l'avancement d'une plus grande justice sociale pour les peuples autochtones au Canada quand «suddenly, two beautiful white women come along, and everybody listens.» Comme le dit la chercheure Mi'kmaw Marie Battiste «Nothing about us without us». En utilisant notre voix, sommes reconnaissante d'avoir eu l'opportunité de rejoindre un auditoire plus vaste et de créer un espace où ceux qui possèdent les savoirs anciens pouvaient partager leurs connaissances traditionnelles.

Quelques années plus tard, nous commençons à prendre conscience de l'impact qu'a pu avoir Projet Traité $\mathrm{n}^{\circ} 4$ dans la vie de nos élèves. Ces élèves sont maintenant adultes et il nous est arrivé à quelques occasions de les rencontrer de façon informelle. À notre grande surprise, ces derniers nous ont parlé encore de comment ils ont été touché par les témoignages des présentateurs lors de la conférence jeunesse. Le fait d'avoir entendu des histoires authentiques provenant $d$ 'individus qui les avaient vécues semble avoir eu un impact profond sur la vie et sur la façon dont ils comprennent le monde qui les entourent. Nous avons aussi pris conscience de l'importance d'établir et d'entretenir les relations avec nos conseillers autochtones. La création de liens d'amitié avec ces derniers est très importante et constitue la pierre angulaire de ce projet éducatif car sans ces liens Projet Traité no 4 ne pourrait pas avoir la même authenticité. L'établissement de ces relations prennent du temps et nous avons 
compris au fil du temps qu'il était important d'être patient et de prendre son temps afin que chaque étape du processus soit réalisée de façon respectueuse.

En somme, tel que mentionné dans le programme sur l'étude des Traités en Saskatchewan, nous croyons que ce projet éducatif a permis à nos élèves de comprendre de façon plus concrète l'expression «Nous appartenons tous aux traités». En tant qu'enseignantes, nous avons eu aussi l'occasion, dans une classe d'immersion française en milieu minoritaire, d'utiliser une approche multidisciplinaire afin de favoriser l'apprentissage des thèmes reliés à la réconciliation. Notre désir était de permettre à nos élèves d'entrer en relation avec des membres des peuples autochtones qui résident sur le territoire du Traité $n^{\circ} 4$. Pour ce faire, nous comprenons que la langue anglaise, qui représente la langue de la majorité, puisse avoir prépondérance lors de ces rencontres. Cependant, nous croyons que notre rôle en tant qu'enseignantes d'immersion française consiste à favoriser un contexte d'enseignement qui encourage une conscientisation au niveau de l'utilisation de la langue française, et ce, malgré la complexité des idées qui sont présentées. Notre désir est de permettre à nos élèves de développer une meilleure compréhension des enjeux reliés à une histoire nationale plus inclusive, tout en favorisant l'apprentissage de la langue française en milieu minoritaire.

\section{NOTES}

1. Les auteures aimeraient remercier le Conseil des Arts de la Saskatchewan, l'Université des Premières Nations du Canada et la Fondation McDowell pour le soutien financier qui a été reçu pour ce projet éducatif, l'ainé Nehiyaw, Noel Starblanket, Calvin Racette, Sandra Bellegarde, Ray Keighley, Dr. Angelina Weenie (Cri des Plaines), Dr. Kathleen O'Reilly, Hillary Ibbott-Neizner, Monique Bowes ainsi que les examinateurs anonymes. Nous sommes reconnaissantes de leur soutien.

2. Plus d'informations au sujet du Projet Traité 4 sont disponibles sur le blog : http://treaty4thenextgeneration.blogspot.com et sur le fil Twitter @Treaty4Project. 


\section{BIBLIOGRAPHIE}

ALLARD, R., LANDRY, R. \& DEVEAU, K. (2005) «Conscientisation ethnolangagière et comportement engagé en milieu minoritaire», Francophonies d'Amérique, n 20, p. 95-109.

B A L LINGER, S., LYSTER, R., STERZUK, A. et GENESEE F. (2017) «Context-appropriate cross linguistic pedagogy. Considering the role of language status in immersion education», Journal of Immersion and Content-Based Language Education. vol. 5, $\mathrm{n}^{\circ} 1$, p. 30-57.

BOURDIEU, P. (2001) Langage et pouvoir symbolique, Paris, Seuil.

BOUTOUCHENT, F. (2016) «Le passage du milieu francophone minoritaire au milieu francophone majoritaire: étude d'une expérience d'enseignants en formation pour comprendre l'influence du milieu sur le développement professionnel». Revue Canadienne de linguistique appliquée: vol. 19, no 1, p. 84108.

COMMISSION DE VÉRITÉ ET RÉCONCILIATION DU CANADA (2015) Pensionnats du Canada: La réconciliation: Rapport final de la Commission de vérité et réconciliation du Canada (vol. 6), McGill-Queen's Press-MQUP.

CUMMIN, J. (2014) «Rethinking pedagogical assumptions in Canada French immersion programs», Journal of Immersion and Content-Based Language Education, vol. 2, n 1, p. 3-22.

D ASCHUK, J. W. (2013) Clearing the plains: Disease, politics of starvation, and the loss of Aboriginal life, Regina, SK: University of Regina Press.

LAROQUE, E. (2016) «Colonialism Lived» in METCALFECHENAIL, D. (Ed.), In this together: Fifteen stories of truth and reconciliation (p.133-144). Victoria, BC: Brindle \& Glass.

LANDRY, R. (2008) «Au-delà de l'école: le projet politique de l'autonomie culturelle», Francophonies d'Amérique, $\mathrm{n}^{\circ} 26$, p. $147-181$.

(2009) «Autonomie culturelle et vitalité des communautés de langue officielle en situation minoritaire», Revue de la common law en francais, $\mathrm{n}^{\circ} 11$, p. 19-43.

(2015) «Légitimité et devenir en situation linguistique minoritaire.» Linguistic Minorities and Society, n 5, p. 58-83.

LANDRY, R., et ALLARD, R. (2016) «Bilinguisme et construction identitaire d'élèves d'écoles de langue anglaise au Québec» Minorités linguistiques et société/Linguistic Minorities and Society, $\mathrm{n}^{\circ} 7, \mathrm{p} .18-47$. 
LYSTER, R. (2017) Content-based Language teaching. Routledge.

(2007) Learning and teaching languages through content: A counterbalanced approach (Vol. 18). John Benjamins Publishing.

MCINTOSH, P. (1990) «White privilege and male privilege: a personal account of coming to see correspondences through work in women's studies 1988», Independent school [Reprinted excerpt from Working Paper 189]. The Wellesley College Center for Research on Women. Winter.

OFFICE OF THE TREATY COMMISSIONER (2014) Treaty Backgrounder: Overview of Treaties in Saskatchewan. Repéré: http://www.otc.ca/public/uploads/resource_photo/Treaty_ Backgrounder.pdf

PETE, S. Schneider, B. O’Reilly, K. (2013). «Decolonizing our Practice Indigenizing Our Teaching», First Nations Perspectives, vol. 5, $\mathrm{n}^{\circ} 1$, p. 99-115.

SASKATCHEWAN EDUCATION (2013). Treaty Education: Outcomes and indicators. Repéré de_https://www.edonline. sk.ca/bbcswebdav/library/materials/english/docs/Treaty $\% 20$ Education $\% 20$ Outcomes $\% 20 \% 26 \% 20$ Indicators $\% 20$ \%20Feb\%2021\%202013.pdf

SKUTNABB-KANGAS (2000) «Linguistic Genocide in Education - Or Worldwide Diversity and Human Rights?», Mahwah, NJ: Lawrence Erlbaum Associates.

STEYAERT, C., OSTENDORP., et GAIBROIS., C. (2011) «Multilingual organizations as 'linguascapes': Negociating the position of English through discursive practices», Journal of World Business, $\mathrm{n}^{\circ} 46$, p. 270-278.

SWAIN, M., et LAPKIN, S. (2013) «A Vygotskian sociocultural perspective on immersion education: The L1/L2 debate», Journal of Immersion and Content-Based Education, $\mathrm{n}^{\circ} 1, \mathrm{p} .101-$ 129.

SIMPSON, L. (2011) Dancing on our turtle's back: Stories of Nishnaabeg re-creation, resurgence and a new emergence, Winnipeg, MB: Arbeiter Ring.

TUCK, E., et YANG, K. W. (2012) «Decolonization is not a metaphor», Decolonization: Indigeneity, Education \& Society, vol. 1, $\mathrm{n}^{\circ}$ 1, http://decolonization.org/index.php/des/article/view/18630

VÉZINA, M., et HOULE, R. (2014) «La transmission de la langue française au sein des familles exogames et endogames francophones au Canada», Cahiers québécois de démographie, vol. $43, \mathrm{n}^{\circ} 2$, p. 399-438. 
VON STADEN, A., et STERZUK, A. (2017). «Un-frenching» des Canadiennes françaises: histoires des Fransaskoises en situation linguistique minoritaire» Canadian Journal of Applied Linguistics/Revue canadienne de linguistique appliquée, vol. 20, $\mathrm{n}^{\circ} 1$, p. 98-114.

WEENIE, A. (2014) "Reflections on teacher as change agent in indigenous education», in THOMAS, L. (Ed.), Becoming teacher: Sites for teacher development in Canadian Teacher Education (p. 503-522). Canadian Association for Teacher Education.

WILSON, S. (2001) «What is an indigenous research methodology?» Canadian Journal of Native Education, vol. 25, n 2, p. 175-179.

$* * * * * * * * * *$

Naomi Fortier-Fréçon détient une maîtrise en enseignement de l'histoire de l'Université de Sherbrooke (Québec). Au cours des dix dernières années, elle a travaillé comme enseignante de sciences sociales dans le programme d'immersion française du conseil scolaire des écoles publiques de Régina. Leia Laing travaille en milieu minoritaire francophone depuis plus de dix ans. Elle est enseignante au conseil des écoles fransaskoises et s'intéresse au développement de stratégies pédagogiques qui permettent de développer une meilleure compréhension des enjeux reliés à la justice sociale. En 2017, Naomi et Leia ont été lauréates du Prix d'histoire du Gouverneur général pour l'excellence en enseignement. 